\title{
Propagation of a spherical wave in elastoplastic medium with complex equations of state
}

\author{
Sherzod Khudainazarov ${ }^{1 *}$, Burkhon Donayev ${ }^{2}$, and $B$ Ashirov ${ }^{2}$ \\ ${ }^{1}$ Tashkent Institute of Irrigation and Agricultural Mechanization Engineers, \\ Tashkent, Uzbekistan \\ ${ }^{2}$ Karshi Engineering-Economics institute, Karshi, Uzbekistan
}

\begin{abstract}
The propagation of a spherical wave in the soil is solved in an analytically inverse way for soils with more complex equations of state. The results are obtained to propagate a spherical shock wave in soil with a more complex equation of state for the shape change in the medium. The study shows that taking into account the nonlinear elastic shock waves of the annular stress leads to an increase compared to the elastic medium. Note that in using a complicated equation of state of the soil, a spherical shock wave propagates in the soil, behind the front of which, in the disturbance region, the medium is unloaded.
\end{abstract}

\section{Introduction}

A shock wave is an area of compression of a medium, which in the form of a spherical layer propagates at a supersonic speed in all directions from the source of its formation. Depending on the medium in which the shock wave propagates (in air, water or soil), it is respectively called an air shock wave, a shock wave in water, a seismic explosion wave in the ground.

Distinguish between a shock wave of natural and anthropogenic origin. Natural waves include shock waves caused by volcanic eruptions, earthquakes, hurricanes, tornadoes, falling meteorites, etc. The anthropogenic shock waves are those that occur as a result of explosions of nuclear devices, chemical explosions, explosions at nuclear power facilities, explosions at oil refining and petrochemical industries, explosions of gas-air mixtures or mixtures of flammable liquids and gases with the air. Most of the destruction and damage to buildings and structures, equipment of industrial facilities, and damage to people, as a rule, is caused by the action of a shock wave.

In [1-2], a methodology for three-dimensional reconstruction of the position and shape of the blast shock wave as a function of time was developed. A series of blasting tests is performed, during which the blasting process is displayed by several high-speed digital cameras scattered over a large area. High speed images are processed using the Schlieren method for shock wave imaging. Shock propagation is measured and corresponds to the Dewey equation. Analysis of the position and propagation of the shock wave makes it

* Corresponding author: scherzodshox77@mail.ru 
possible to reveal asymmetries at the shock front due to the asymmetric explosion process. The methods developed here are shown to be useful tools that can be implemented to complement traditional point tools in current explosive research testing and provide improved explosion performance over traditional field testing methods.

In [3-6], the propagation of a strong spherical shock wave in a dusty gas with or without self-gravity effects in the case of isothermal and adiabatic flows was investigated. It is assumed that the dusty gas is a mixture of fine solid particles and ideal gas. Analyzes show that after the effects of the gravitational field are turned on, the impact force unexpectedly increases, and noticeable differences in the distribution of flux variables are found. Increasing the time also increases the power of the blow. It is investigated that taking into account the isothermal flow increases the impact force and eliminates the singularity in the density distribution.

In [7-11], nonlinear relations based on the laws of Winkler and Coulomb are proposed to describe the process of shear interaction between structures and soil. Their advantages and disadvantages are shown, as well as the suitability of the experimental results. And also, one-dimensional non-stationary wave problems for soil and structure are numerically solved using the method of characteristics and the method of finite differences. Analysis of the obtained numerical solutions shows a significant dependence of longitudinal stresses on wave processes in the soil, the dynamic stress state of the soil and the mechanical properties of the soil and the material of the structure. The results obtained are the basis for developing a new standard calculation of the strength of underground engineering structures under seismic impact.

In works [12-15], studies were carried out to analyze the behavior of a high-rise structure with various kinematic effects, taking into account the real geometry, dissipative and nonlinear properties of the structure material. A generalized approach to the dynamic calculation of high-rise structures has been developed, frequency characteristics have been constructed at various points of the structure. It was found that the nonlinear properties of the material of structures appear when the impact of a spherical wave can cause significant deformations in the structure. This applies not only to the magnitude of the impact force but also to its frequency content. Suppose nonlinear elastic deformation of the material manifests itself in the structure. In that case, this leads to a decrease in the displacement amplitudes of the points and an increase in the oscillation period compared to a linear elastic structure with similar kinematic effects.

In [16], the problem of the propagation of a spherical shock wave in an elastoplastic medium is solved analytically and numerically by the method of characteristics. The results show that taking into account the diagrams of nonlinear elastic impact leads to an increase in the circular stress wave as compared to the elastic medium. It turned out that the concentration of stresses on a spherical cavity is higher than on a cylindrical one.

In [17-19], a variational statement, methods and algorithms for solving various dynamic problems for a viscoelastic system are given, taking into account the conditions of nonreflection on the boundary of a finite base. The dynamic behavior of an inhomogeneous viscoelastic system under a short-term intense load on the foundation is investigated. A weak dependence of energy dissipation in a system with hereditary viscoelastic properties of a material on the frequency of natural vibrations is revealed and a dependence of the wave removal of energy on the main natural frequencies of the system vibrations.

In [20-21], the propagation of a spherical wave in linear-elastic and viscoelastic media was investigated. Here, a new model and a new approach to the analytical solution of the problem have been developed to model viscoelastic damping. Wave propagation is achieved by cascading separate geometric and viscoelastic damping mechanisms. Comparison of the analytical model with the results of dynamic finite element modeling 
shows that the method of cascading individual transfer functions is a suitable approach for wave propagation in viscoelastic media.

In [22-23], the propagation of axisymmetric viscoelastic waves in extended multilayer cylindrical structures was investigated. The classical methods of mathematical physics are used to solve boundary value problems in a cylindrical coordinate system, the spatial Fourier transform, the method of complex amplitudes for the variable components of the displacement vector and the stress tensor. The low-frequency resonances of the cylindrical shell are investigated, and the physical regularities of the formation of the pitch of the sound of its fundamental tone are determined.

Dynamic analysis and the results of engineering analysis on the nature of the operation of structures during strong earthquakes indicate that the rigidity of structures does not always remain constant. Therefore, the parameters of the current response of structures must be determined only with the help of nonlinear analysis, which allows developing more reasonable design and construction methods, increasing the efficiency of structures while maintaining the required level of reliability. However, the problem under consideration is three-dimensional and non-stationary; therefore, methods for calculating underground structures for dynamic effects due to the complexity of the physical and mechanical properties of the soil, the nature of seismic and seismic explosive effects, the shape and geometry of structures have not yet been developed enough. In this direction, a certain success has been achieved with an obstacle of various shapes within the framework of the linear theory of elasticity. Still, a limited number of works have been devoted to elastoplastic deformations.

In this regard, in this work, several specific problems have been solved in relation to studying the interaction of waves with a spherical cavity in soils, taking into account their complex elastoplastic deformations.

The main goal of this article is to study the one-dimensional and two-dimensional nonstationary problem of the dynamic theory of plasticity as applied to the calculation of the parameters of the medium in cases of propagation and deformation of waves from different surfaces, based on the deformation theory with more complex equations of state.

This article aims to study the one-dimensional and two-dimensional nonstationary problem of the dynamic theory of plasticity as applied to the calculation of the parameters of the medium in cases of propagation and deformation of waves from various surfaces, based on the deformation theory with more complex equations of state.

\section{Methods}

In this work, the problem of the propagation of a spherical wave in the soil is solved in an analytically inverse way for soils with more complex equations of state. The complexity of the equations of state is based on the assumption that the shape change function (stress intensity) of the deformation theory [24-25], which is one of the equations of state of the medium, depends on the first invariants of the strain tensor, i.e. This function in the process of loading the soil (Fig.1), according to the experimental data [26], is represented as:

$$
\begin{gathered}
\sigma_{i}=\left(\varepsilon, \varepsilon_{i}\right)=\sigma_{i}^{H}\left(\varepsilon_{i}\right)-\frac{\sigma(\varepsilon)+25}{15}\left[\sigma_{i}^{b}\left(\varepsilon_{i}\right)-\sigma_{i}^{H}\left(\varepsilon_{i}\right)\right], \\
\sigma_{i}^{H, b}=a_{j}\left[1-\exp \left(-b_{j} \varepsilon_{i}\right)\right]+c_{j} \varepsilon_{i}, j=1,2,
\end{gathered}
$$


In formulas (2), the coefficients $a_{j}, b_{j}, c_{j}$ are assumed to be known constant values. For the function $\sigma(\varepsilon)$ included in (1), we have the expression

$$
\sigma(\varepsilon)=\left(\alpha_{1}+\alpha_{2}|\varepsilon|\right) \varepsilon, \quad \sigma_{i}\left(\varepsilon_{i}\right)=\left(\beta_{1}+\beta_{2} \varepsilon_{i}\right) \varepsilon_{i}
$$
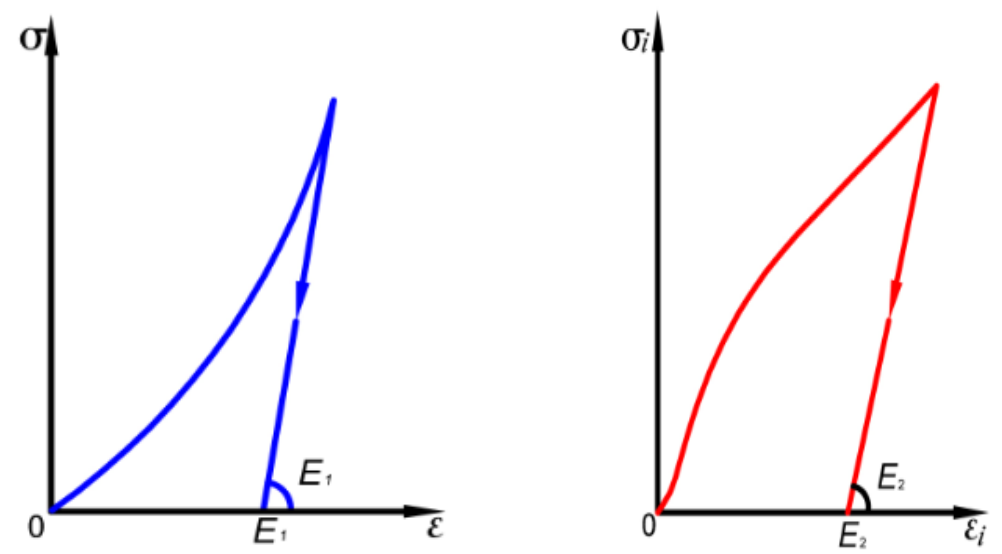

Fig. 1. Change of the first and second invariants of the stress tensors $\sigma, \sigma_{i}$ and deformation $\varepsilon, \varepsilon_{1}$
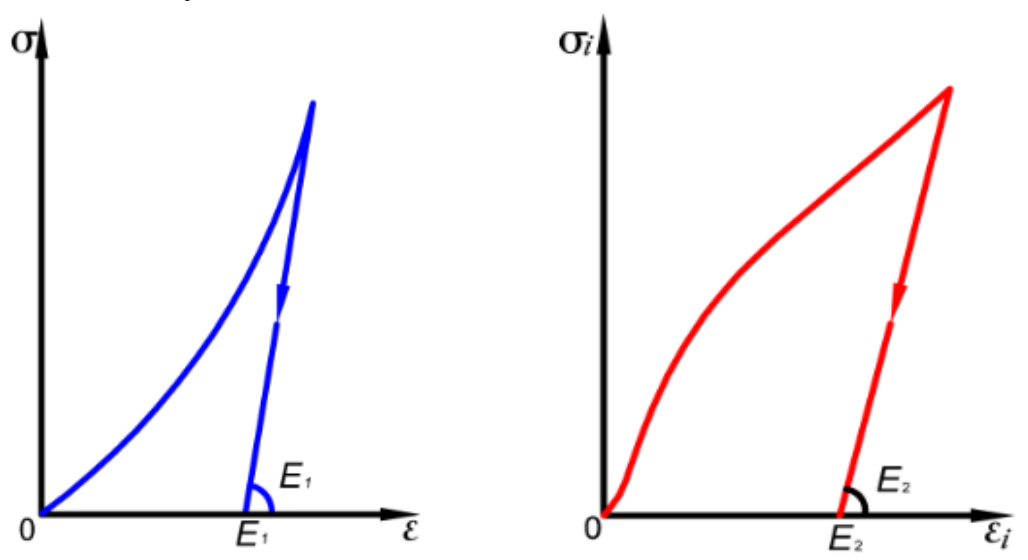

Fig. 1. Change of the first and second invariants of the stress tensors $\sigma, \sigma_{i}$ and deformation $\varepsilon, \varepsilon_{1}$ 


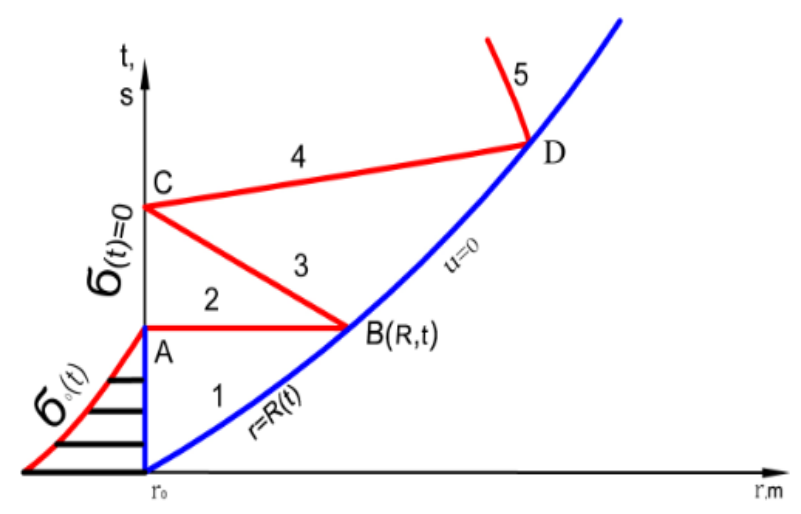

Fig. 2. Graph of the change in the shock wave in the ground $r=R(t)$

In the process of solving the problem, it is assumed that when using

$$
\rho_{0} \frac{\partial^{2} u}{\partial t^{2}}=\frac{\partial \sigma_{r r}}{\partial r}+2 \frac{\left(\sigma_{r r}-\sigma_{\varphi \varphi}\right)}{r}
$$

and (1), a spherical shock wave propagates in the soil (Fig.2), at the front of which the medium is instantly loaded in a nonlinear manner, followed by a linear irreversible unloading with Young's moduli $E_{1}$ and $E_{2}$. This assumption is confirmed by the solution and numerical calculations of the problem. For the analytical construction of the solution to the problem, the surface of the shock wave $r=R(t)$, as in (1), is given as a polynomial of the second degree with respect to time, and the load profile $\sigma_{0}(t)$ is determined from the solution of the problem. Then from the condition

$$
\begin{aligned}
& \sigma_{r r}^{*}=-\rho_{0} \dot{R}(t) u_{t}^{*}, u_{t}^{*}=-R(t) \varepsilon_{r r}^{*}, \\
& u(r, t)=\mathrm{O} \text { at } r=R(t)
\end{aligned}
$$

taking into account the first equation (3), (4) and (1), all the parameters of the medium, including the mass velocity $u^{*}$ and deformation $\varepsilon^{*}=\varepsilon_{r r}^{*}$ (since at the mixing wave front $u^{*} \equiv 0$ ) at the shock wave front, are obtained by the known quantities depending on time $t$ or coordinates $r$. In this case, to determine the deformation $\varepsilon^{*}(t)$ at the shock front, instead of the first equation,

$$
\begin{aligned}
& \varepsilon^{*}(t)=\varepsilon_{r r}(t)=-\frac{\rho_{0} \dot{R}^{2}(t)-\left(\alpha_{1}+\frac{4}{9} \beta_{1}\right)}{\left(\alpha_{2}-\frac{8}{27} \beta_{2}\right)}, \\
& u_{t}^{*}=-\dot{R}(t) \varepsilon^{*}(t),
\end{aligned}
$$


we obtain a transcendental equation of the form, which is solved numerically using the standard procedure.

$$
\begin{aligned}
& \alpha_{1}-\alpha_{2} \varepsilon+\frac{4}{9}\left\{a_{2}\left[1+\frac{\left(\alpha_{1} \varepsilon-\alpha_{2} \varepsilon^{2}+25\right)}{15}\right] \frac{\left[1-\exp \left(-b_{2} \varepsilon_{i}\right)\right]}{\varepsilon_{1}}+\right. \\
& +\left[c_{2}-\left(c_{1}-c_{2}\right) \frac{\alpha_{1} \varepsilon-\alpha_{2} \varepsilon^{2}+25}{15}\right]-a_{1} \frac{\alpha_{1} \varepsilon-\alpha_{2} \varepsilon^{2}+25}{15 \varepsilon_{i}} . \\
& \left.\cdot\left[1-\exp \left(-b_{2} \varepsilon_{i}\right)\right]\right\}-\rho_{0} R^{2}(t)=0, \text { where }_{i}=-\frac{2}{3} \varepsilon_{r r}, \varepsilon_{r r}=\varepsilon \prec 0,
\end{aligned}
$$

Let equation (7) have a solution

$$
\varepsilon=\varepsilon^{*}(t)
$$

If the equations of the shock wave surface $r=r_{0}+R_{1} t-R_{2} t^{2} / 2$ are represented in the form $t=t(r)$, then the wave velocity is written relative to the coordinate and from (7), we find

$$
\varepsilon=\varepsilon^{*} r
$$

Thus, by analogy with (6), at the front of a spherical shock wave, we have the following conditions:

$$
\frac{\partial u}{\partial r}=\varepsilon^{*}(t), \frac{\partial u}{\partial t}=-R(t) \cdot \varepsilon^{*}(t)
$$

In this case, in the region of perturbation, the equation

$$
\frac{\partial^{2} u}{\partial t^{2}}=a_{0}^{2}\left[\frac{\partial^{2} u}{\partial r^{2}}+\frac{2}{r}\left(\frac{\partial u}{\partial r}-\frac{u}{r}\right)+\frac{Q(r)}{\rho_{0} a_{0}^{2}}\right]
$$

with decision

$$
\begin{aligned}
& u(r, t)=\frac{\psi^{\prime}\left(r-a_{0} t\right)+\varphi^{\prime}\left(r+a_{0} t\right)}{r}-\frac{\psi\left(r-a_{0} t\right)+\varphi\left(r+a_{0} t\right)}{r^{2}}- \\
& \frac{r}{3\left(\lambda+2 G_{0}\right)} \int_{r_{0}}^{r} Q(r) d r+\frac{1}{3\left(\lambda+2 G_{0}\right) r^{2}} \int_{r_{0}}^{r} Q(r) r^{3} d r
\end{aligned}
$$

where $\psi$ and $\phi$ are unknown functions.

Here is the function $Q(r)$, expressed by the formula 


$$
\begin{aligned}
& Q(r)=\frac{\partial}{\partial r}\left[\sigma_{r r}(r)-\rho_{0} a_{0}^{2} \varepsilon^{*}(r)\right]+ \\
& +\frac{2}{r}\left[\sigma_{r r}^{*}(r)-\sigma_{\phi \phi}(r)-2 G \varepsilon^{*}(r)\right], \\
& \rho_{0} a_{0}^{2}=\lambda+2 G_{0} ; \quad \lambda_{0}=E_{1}-\frac{2}{9} E_{2} ; \\
& G_{0}=\frac{1}{3} E_{2} ; \quad a_{0}=\sqrt{\left(E_{1}+\frac{4}{9} \beta_{1}\right) / \rho_{0}}
\end{aligned}
$$

directly depends on the solution of the transcendental equation (1.7), represented in the form (1.9). To find the unknown functions $\psi$ and $\phi$ included in (1.12), substituting (1.12) into (1.10), we obtain a system of two equations in the form:

$$
\begin{aligned}
& \frac{\psi^{\prime \prime}\left(R-a_{0} t\right)+\phi^{\prime \prime}\left(R+a_{0} t\right)}{R(t)}-\frac{1}{\left(\lambda_{0}-2 G_{0}\right)} \int_{r_{0}}^{R(t)} Q(\xi) d \xi-\frac{1}{\left(\lambda_{0}-2 G_{0}\right)} \\
& \cdot\left\{\left[\sigma_{r r}(R(t))-\rho_{0} a_{0}^{2} \varepsilon^{*}(R(t))\right]-\left[\sigma_{r r}\left(r_{0}\right)-\rho_{0} a_{0}^{2} \varepsilon^{*}\left(r_{0}\right)\right]\right\}=\varepsilon^{*}(t) \\
& 2 R(t) \psi^{\prime \prime}\left(R-a_{0} t\right)-\left[\psi^{\prime}\left(R-a_{0} t\right)-\varphi^{\prime}\left(R+a_{0} t\right)\right]- \\
& -\frac{R^{2}(t)}{\left(\lambda_{0}+2 G_{0}\right)} \cdot \cdot \int_{r_{0}}^{R(t)} Q(\xi) d \xi- \\
& -\frac{R^{2}(t)}{\left(\lambda_{0}+2 G_{0}\right)}\left\{\begin{array}{l}
{\left[\sigma_{r r}^{*}(R(t))-\rho_{0} a_{0}^{2} \varepsilon^{*}(R(t))\right]-} \\
-\left[\sigma_{r r}\left(r_{0}\right)-\rho_{0} a_{0}^{2} \varepsilon^{*}\left(r_{0}\right)\right]
\end{array}\right\}= \\
& =R^{2}(t)\left(1+R(t) / a_{0} \varepsilon^{*}(t)\right)
\end{aligned}
$$

Now, substituting (15) into (14), after some transformations, we have:

$$
\begin{aligned}
& \psi^{\prime \prime}(z)=\frac{1}{2}\left\{\frac{1}{\left(\lambda_{0}+2 G_{0}\right)} \int_{r_{0}}^{R(F(z))} Q(\xi) d \xi+\frac{R\left(F_{1}(z)\right)}{\left(\lambda_{0}+2 G_{0}\right)} \cdot \frac{R\left(F_{1}(z)\right)}{\left[R\left(F(z)-a_{0}\right]\right.} .\right. \\
& \cdot Q\left[R\left(F_{1}(z)\right)\right]+\frac{1}{\left(\lambda_{0}+2 G_{0}\right)}\left[\sigma_{r r}^{*}\left(R\left(F_{1}(z)\right)\right)-\rho_{0} a_{0}^{2} \varepsilon^{*}\left(R\left(F_{1}(z)\right)\right)\right]- \\
& -\frac{1}{\left(\lambda_{0}+2 G_{0}\right)}\left[\sigma_{r r}^{*}\left(r_{0}\right)-\rho_{0} a_{0}^{2} \varepsilon^{*}\left(r_{0}\right)\right]+\frac{R\left(F_{1}(z)\right) \cdot R\left(F_{1}(z)\right)}{\left(\lambda_{0}+2 G_{0}\right)\left[R\left(F_{1}(z)\right)-a_{0}\right]} \cdot \\
& \cdot\left[\sigma_{r r}^{*}\left(R\left(F_{1}(z)\right)\right)-\rho_{0} a_{0}^{2} \varepsilon^{*}\left(R\left(F_{1}(z)\right)\right)\right]+\frac{2 R\left(F_{1}(z)\right)-a_{0}}{R\left(F_{1}(z)\right)-a_{0}}\left[1+R\left(F_{1}(z)\right) / a_{0}\right] . \\
& \cdot \varepsilon^{*}\left(F_{1}(z)\right)+\frac{R\left(F_{1}(z)\right) \cdot R\left(\ddot{F}_{1}(z)\right)}{a_{0}\left[R\left(F_{1}(z)\right)-a_{0}\right]} \varepsilon^{*}\left(F_{1}(z)\right)+ \\
& \left.+\frac{R\left(F_{1}(z)\right)}{R\left(F_{1}(z)\right)-a_{0}}\left[1+R\left(F_{1}(z)\right) / a_{0}\right] \varepsilon^{*}\left(F_{1}(z)\right)\right\}
\end{aligned}
$$


Where $F_{1}(z)$ is the root of the equation $R-a_{0} t=z$ with respect to $t$. Denoting the right-hand side of (16) through functions $\phi(z)$ and appropriately substituting (16) into (15), we obtain an expression for the desired function $\phi\left(r+a_{0} t\right)$.. Then, taking with considering (12), the solution of the spherical problem, in this case, is represented as:

$$
\begin{aligned}
& u(r, t)=\frac{1}{r}\left\{\int_{R-a_{0} t}^{r-a_{0} t} d \xi \xi_{1} \int_{z_{10}}^{\xi} \phi(\xi) d \xi-\int_{R+a_{0} t}^{r+a_{0} t} d \xi_{1}^{R\left(F_{2}(\xi)\right)-a_{0} F_{2}(\xi)} \int_{z_{10}} \phi(\xi) d \xi+\right. \\
& +\int_{R+a_{0} t}^{r+a_{0} t} \frac{R\left(F_{2}(\xi)\right)}{\lambda_{\mathrm{o}}+2 G_{\mathrm{O}}} d \xi_{1} \int_{r_{0}}^{R\left(F_{2}(\xi)\right)} Q(\xi) d \xi+\int_{R+a_{0} t}^{r+a_{0} t} R\left(F_{2}(\xi)\right)\left[\sigma_{r r}^{*}\left(R\left(F_{2}(\xi)\right)\right)-\right. \\
& \left.-\rho_{0} a_{0}^{2} \varepsilon^{*}\left(R\left(F_{2}(\xi)\right)\right)\right] d \xi-\int_{R+a_{0} t}^{r+a_{0} t} \frac{R\left(F_{2}(\xi)\right)}{\lambda_{0}+2 G_{0}}\left[\sigma_{r r}\left(r_{0}\right)-\rho_{0} a_{0}^{2} \varepsilon^{*}\left(r_{0}\right)\right] d \xi+ \\
& \left.+\int_{R+a_{0} t}^{r+a_{0} t} R\left(F_{2}(\xi)\right) \cdot \varepsilon^{*}\left(F_{2}(\xi)\right) d \xi\right\}-\frac{1}{r^{2}}\left\{\int_{R-a_{0} t}^{r-a_{0} t} d \xi_{2} \cdot \int_{z_{10}}^{\xi} d \xi_{1} \cdot \int_{z_{10}}^{\xi} \phi(\xi) d(\xi)-\right. \\
& -\int_{R+a_{0} t}^{r+a_{0} t} d \xi_{2} \int_{z_{20}}^{\xi_{2}} d \xi_{1} \int_{z_{10}}^{R\left(F_{2}(\xi)\right)-a_{0} F_{2}(\xi)} \phi(\xi) d \xi+\int_{R+a_{0} t}^{r+a_{0} t} d \xi_{2} \int_{z_{20}}^{\xi_{2}} \frac{R\left(F_{2}(\xi)\right)}{\lambda_{0}+2 G_{0}} d \xi_{1} \int_{r_{0}}^{R\left(F_{2}\left(\xi_{1}\right)\right)} Q(\xi) d \xi+ \\
& +\int_{R+a_{0} t}^{r+a_{0} t} d \xi_{1} \int_{z_{20}}^{\xi_{1}} \frac{R\left(F_{2}(\xi)\right)}{\lambda_{0}+2 G_{0}} \cdot\left[\sigma_{r r}^{*}\left(R\left(F_{2}(\xi)\right)\right)-\rho_{0} a_{0}^{2} \varepsilon^{\prime \prime}\left(R\left(F_{2}(\xi)\right)\right)\right] d \xi-\int_{R+a_{0} t}^{r+a_{0} t} d \xi_{2} \int_{z_{20}}^{\xi_{1}} \frac{R\left(F_{2}(\xi)\right)}{\lambda_{0}+2 G_{0}} d \xi . \\
& \left.\cdot\left[\sigma_{r r}\left(r_{0}\right)-\rho_{0} a_{0}^{2} \varepsilon^{*}\left(r_{0}\right)\right] d \xi+\int_{R+a_{0} t}^{r+a_{0} t} d \xi_{1} \int_{z_{20}}^{\xi_{1}} R\left(F_{2}(\xi)\right) \cdot \xi^{*}(F(\xi)) d \xi\right\}+\frac{1}{2}\left(\frac{1}{R(t)}-\frac{1}{r}\right)\left\{C_{35}^{+}-C_{24}^{-} a_{0} t+\right. \\
& +\int_{z_{10}}^{R(t)-a_{0} t} d \xi_{2} \int_{z_{10}}^{\xi_{2}} d \xi_{1} \int_{z_{10}}^{\xi_{1}} \phi(\xi) d \xi-\int_{z_{20}}^{R(t)-a_{0} t} d \xi_{2} \int_{z_{20}}^{\xi_{2}} d \xi_{1} \int^{R\left(F_{2}\left(\xi_{1}\right)\right)-a_{0} F_{2}\left(\xi_{1}\right)} \phi(\xi) d \xi\left(\int_{z_{20}}^{R(t)+a_{0} t} d \xi_{2} \int_{z_{20}}^{\xi_{2}} \frac{R\left(F_{2}\left(\xi_{1}\right)\right)}{\lambda_{0}+2 G_{0}} d \xi_{1}\right. \\
& \cdot \int_{r_{0}}^{R\left(F_{2}\left(\xi_{1}\right)\right)} Q(\xi) d \xi+\int_{z_{20}}^{R(t)+a_{0} t} d \xi_{1} \int_{z_{20}}^{\xi_{1}} \frac{R\left(F_{2}(\xi)\right)}{\lambda_{0}+2 G_{0}} \cdot\left[\sigma_{r r}^{*}\left(R\left(F_{2}(\xi)\right)\right)-\rho_{0} a_{0}^{2} \varepsilon^{*}\left(R\left(F_{2}(\xi)\right)\right)\right] d \xi- \\
& \left.-\int_{z_{20}}^{R(t)+a_{0} t} d \xi_{1} \int_{z_{20}}^{\xi_{1}} \frac{R\left(F_{2}(\xi)\right)}{\lambda_{0}+2 G_{0}} \cdot\left[\sigma_{r r}^{*}\left(r_{0}\right)-\rho_{0} a_{0}^{2} \varepsilon^{*}\left(r_{0}\right)\right] d \xi+\int_{z_{20}}^{R(t)+a_{0} t} d \xi_{1} \int_{z_{10}}^{\xi_{1}} R\left(F_{2}(\xi)\right) \cdot \varepsilon^{*}\left(F_{2}(\xi)\right) d \xi\right\}+ \\
& +\frac{1}{r}\left\{\frac{R^{2}(t)}{3\left(\lambda_{0}+2 G_{0}\right)} \int_{r_{0}}^{R(t)} Q(\xi) d \xi-\frac{1}{3\left(\lambda_{0}+2 G_{0}\right) R(t)} \int_{r_{0}}^{R(t)} \xi^{3} Q(\xi) d \xi+\frac{1}{\lambda_{0}+2 G_{0} R(t)} .\right. \\
& \left.\cdot \int_{r_{0}}^{R(t)} \xi^{2}\left[\sigma_{r r}(\xi)-\rho_{0} a_{0}^{2} \varepsilon^{*}\left(r_{0}\right)\right] d \xi-\frac{R^{2}(t)}{3\left(\lambda_{0}+2 G_{0}\right)}\left[\sigma_{r r}\left(r_{0}\right)-\rho_{0} a_{0}^{2} \varepsilon^{*}\left(r_{0}\right)\right]\right\}+\frac{r_{0}^{3}}{3\left(\lambda_{0}+2 G_{0}\right) \cdot R(t)} \cdot \\
& \left.\cdot\left[\sigma_{r r}^{*}\left(r_{0}\right)-\rho_{0} a_{0}^{2} \varepsilon^{*}\left(r_{0}\right)\right]\right\}-\frac{r}{3\left(\lambda_{0}+2 G_{0}\right)} \int_{r_{0}}^{r} Q(\xi) d \xi+\frac{1}{3\left(\lambda_{0}+2 G_{0}\right) r^{2}} \cdot \int_{r_{0}}^{r} \xi^{3} Q(\xi) d \xi-\frac{1}{\left(\lambda_{0}+2 G_{0}\right) r^{2}} . \\
& \cdot \int_{r_{0}}^{r} \xi^{2}\left[\sigma_{r r}^{*}(\xi)-\rho_{0} a_{0}^{2} \varepsilon^{*}(\xi)\right] d \xi+\frac{r}{3\left(\lambda_{0}+2 G_{0}\right)}\left[\sigma_{r r}^{*}\left(r_{0}\right)-\rho_{0} a_{0}^{2} \varepsilon^{*}\left(r_{0}\right)\right]-\frac{r_{0}^{3}}{3\left(\lambda_{0}+2 G_{0}\right) r^{2}} \cdot \\
& \cdot\left[\sigma_{r r}^{*}\left(r_{0}\right)-\rho_{0} a_{0}^{2} \varepsilon^{*}\left(r_{0}\right)\right] \text {, }
\end{aligned}
$$

Where $F_{2}(\xi)$ is the root of the equation with respect to $R(t)+a_{0} t=z$. From (17), taking with considering (10), to determine the mass velocity $\boldsymbol{U}_{t}$ and the radial component of the soil deformation $\mathcal{E}_{r r}$, we obtain the expressions: 


$$
\begin{aligned}
& u_{t}(r, t)=u_{t}^{*}(r, t(r))+\frac{a_{0}}{r}\left\{-\int_{r-a_{0} t(r)}^{r-a_{0} t} \phi(\xi) d \xi-\int_{R\left(F_{2}\left(r+a_{0} t(r)\right)\right)-a_{0} F_{2}\left(r+a_{0} t(r)\right)}^{R\left(F_{2}\left(r+a_{0} t\right)\right)-a_{0} F_{2}\left(r+a_{0} t\right)}+\frac{R\left(F_{2}\left(r+a_{0} t\right)\right)}{\left(\lambda_{0}+2 G_{0}\right)}\right. \\
& \int_{r_{0}}^{R\left(F_{2}\left(r+a_{0} t\right)\right)} Q(\xi) d \xi-\frac{R\left(F_{2}\left(r+a_{0} t(r)\right)\right.}{\left(\lambda_{0}+2 G_{0}\right)} \int_{r_{0}}^{R\left(F_{2}\left(r+a_{0} t(r)\right)\right)} Q(\xi) d \xi+\frac{R\left(F_{2}\left(r+a_{0} t\right)\right)}{\left(\lambda_{0}+2 G_{0}\right)}\left[\sigma_{r r}^{*}\left(R\left(F_{2}\left(r+a_{0} t\right)\right)\right)-\right. \\
& \left.\left.-\rho_{0} a_{0}^{2} \varepsilon^{*}\left(R\left(F_{2}\left(r+a_{0} t\right)\right)\right)\right]-\frac{R\left(F_{2}\left(r+a_{0} t(r)\right)\right.}{\left(\lambda_{0}+2 G_{0}\right)}\left[\sigma_{r r}^{*}\left(R\left(F_{2}\left(r+a_{0} t\right)\right)\right)-\rho_{0} a_{0}^{2} \varepsilon^{*}\left(R\left(F_{2}\left(r+a_{0} t\right)\right)\right)\right)\right]- \\
& -\frac{\left(R\left(F_{2}\left(r+a_{0} t\right)\right)-R\left(F_{2}\left(r+a_{0} t(r)\right)\right)\right.}{\left(\lambda_{0}+2 G_{0}\right)} \cdot\left[\sigma_{r r}^{*}\left(r_{0}\right)-\rho_{0} a_{0}^{2} \varepsilon^{*}\left(r_{0}\right)\right]+R\left(F_{2}\left(r+a_{0} t\right)\right) \cdot \varepsilon^{*}\left(F_{2}\left(r+a_{0} t\right)\right)- \\
& \left.-R\left(F_{2}\left(r+a_{0} t(r)\right)\right) \cdot \varepsilon^{*}\left(F_{2}\left(r+a_{0} t(r)\right)\right)\right\}-\frac{a_{0}}{r^{2}}\left\{-\int_{r-a_{0} t}^{r+a_{0} t} d \xi \cdot \int_{z_{10}}^{\xi} \phi(\xi) d \xi-\int_{r+a_{0} t(r)}^{r+a_{0} t} d \xi_{1} \int_{z_{10}}^{R\left(F_{2}(\xi)-a_{0} F_{2}(\xi)\right.} \phi(\xi) d \xi+\right. \\
& \int_{r+a_{0} t(r)}^{r+a_{0} t} \frac{R\left(F_{2}(\xi)\right.}{\left(\lambda_{0}+2 G_{0}\right)} d \xi \cdot \int_{r_{0}}^{R\left(F_{2}\left(\xi_{1}\right)\right)} Q(\xi) d \xi+\int_{r+a_{0} t(r)}^{r+a_{0} t} \frac{R\left(F_{2}(\xi)\right)}{\left(\lambda_{0}+2 G_{0}\right)}\left[\sigma_{r r}^{*}\left(R\left(F_{2}(\xi)\right)\right)-\rho_{0} a_{0}^{2} \varepsilon^{*}\left(R\left(F_{2}(\xi)\right)\right)\right] d \xi- \\
& \left.-\int_{r+a_{0} t(r)}^{r+a_{0} t} \frac{R\left(F_{2}(\xi)\right.}{\left(\lambda_{0}+2 G_{0}\right)}\left[\sigma_{r r}^{*}\left(r_{0}\right)-\rho_{0} a_{0}^{2} \varepsilon^{*}\left(r_{0}\right)\right] d \xi+\int_{r+a_{0} t(r)}^{r+a_{0} t} R\left(F_{2}(\xi)\right) \varepsilon^{*}\left(F_{2}(\xi)\right) d \xi\right\}, \\
& \varepsilon_{r r}(r, t)=\frac{\partial u}{\partial r}=\varepsilon_{r r}^{*}(R(t), t)+\frac{1}{2}\left\{\int_{z_{10}}^{r-a_{0} t} \phi(\xi) d \xi-\int_{z_{10}}^{R\left(F_{2}\left(r+a_{0} t\right)-a_{0} F_{2}\left(r+a_{0} t\right)\right.} \phi(\xi) d \xi+\frac{R\left(F_{2}\left(r+a_{0} t\right)\right)}{\left(\lambda_{0}+2 G_{0}\right)} \int_{r_{0}}^{R\left(F_{2}\left(r+a_{0} t\right)\right)} Q(\xi) d \xi+\right. \\
& +\frac{R\left(F_{2}\left(r+a_{0} t\right)\right)}{\left(\lambda_{0}+2 G_{0}\right)}\left[\sigma_{r r}^{*}\left(R\left(F_{2}\left(r+a_{0} t\right)\right)\right)-\rho_{0} a_{0}^{2} \varepsilon^{*}\left(R\left(F_{2}\left(r+a_{0} t\right)\right)\right)\right]-\frac{R\left(F_{2}\left(r+a_{0} t\right)\right)}{\left(\lambda_{0}+2 G_{0}\right)} . \\
& \left.\cdot\left[\sigma_{r r}^{*}\left(r_{0}\right)-\rho_{0} a_{0}^{2} \varepsilon^{*}\left(r_{0}\right)\right]+R\left(F_{2}\left(r+a_{0} t\right)\right) \cdot \varepsilon^{*}\left(F_{2}\left(r+a_{0} t\right)\right)\right\}-\frac{1}{R(t)}\left\{\int_{z_{10}}^{R(t)-a_{0} t} \phi(\xi) d(\xi)-\right. \\
& -\int_{z_{10}}^{R\left(F_{2}\left(R(t)+a_{0} t\right)-a_{0} F_{2}\left(R(t)+a_{0} t\right)\right.} \phi(\xi) d(\xi)+\frac{R\left(F _ { 2 } \left(R(t)+a_{0} t\right.\right.}{\left(\lambda_{0}+2 G_{0}\right)} \int_{r_{0}}^{R\left(F_{2}\left(R(t)+a_{0} t\right)\right)} Q(\xi) d \xi+\frac{R\left(F_{2}\left(R(t)+a_{0} t\right)\right)}{\left(\lambda_{0}+2 G_{0}\right)} . \\
& \cdot\left[\sigma_{r r}^{*}\left(R\left(F_{2}(t)+a_{0} t\right)\right)-\rho_{0} a_{0}^{2} \varepsilon^{*}\left(R\left(F_{2}\left(R(t)+a_{0} t\right)\right)\right)\right]-\frac{R\left(F _ { 2 } \left(R(t)+a_{0} t\right.\right.}{\left(\lambda_{0}+2 G_{0}\right)}\left[\sigma_{r r}^{*}\left(r_{0}\right)-\rho_{0} a_{0}^{2} \varepsilon^{*}\left(r_{0}\right)\right]+ \\
& \left.+R\left(F_{2}\left(R(t)+a_{0} t\right)\right) \cdot \varepsilon^{*}\left(F_{2}\left(R(t)+a_{0} t\right)\right)\right\}-\frac{2}{r^{2}}\left\{\int_{z_{10}}^{r-a_{0} t} d \xi_{1} \int_{z_{10}}^{\xi} \phi(\xi) d(\xi)-\int_{z_{20}}^{r+a_{0} t} d \xi_{1} \int_{z_{10}}^{\left.R\left(F_{2}(\xi)\right)-a_{0} F_{2}\left(\xi_{10}\right)\right)} \phi(\xi) d \xi+\right. \\
& +\int_{z_{20}}^{r+a_{0} t} \frac{R\left(F_{2}(\xi)\right.}{\left(\lambda+2 G_{0}\right)} d \xi_{1} \int_{r_{0}}^{R\left(F_{2}\left(\xi_{1}\right)\right)} Q(\xi) d(\xi)+\int_{z_{20}}^{r+a_{0} t} \frac{R\left(F_{2}(\xi)\right.}{\left(\lambda+2 G_{0}\right)}\left[\sigma_{r r}^{*}\left(R\left(F_{2}(\xi)\right)\right)-\rho_{0} a_{0}^{2} \varepsilon^{*}\left(R\left(F_{2}(\xi)\right)\right)\right] d \xi- \\
& -\int_{z_{20}}^{r+a_{0} t} \frac{R\left(F_{2}(\xi)\right)}{\left(\lambda+2 G_{0}\right)}\left[\sigma_{r r}^{*}\left(r_{0}\right)-\rho_{0} a_{0}^{2} \varepsilon^{*}\left(r_{0}\right)\right] d \xi+\int_{z_{20}}^{r+a_{0} t} R\left(F_{2}(\xi)\right) \cdot \varepsilon^{*}\left(F_{2}(\xi)\right) d \xi+\frac{2}{R^{2}(t)}\left\{\int_{z_{10}}^{R(t)-a_{0} t} d \xi_{1} \int_{z_{10}}^{\xi_{1}} \phi(\xi) d \xi-\right. \\
& -\int_{z_{10}}^{R(t)+a_{0} t} d \xi_{1} \cdot \int_{z_{10}}^{\left.R\left(F_{2}\left(\xi_{1}\right)\right)-a_{0} F_{2}\left(\xi_{1}\right)\right)} \phi(\xi) d \xi+\int_{z_{20}}^{R(t)+a_{0} t} \frac{R\left(F_{2}(\xi)\right)}{\left(\lambda_{0}+2 G_{0}\right)}\left[\sigma_{r r}^{*}\left(R\left(F_{2}(\xi)\right)\right)-\rho_{0} a_{0}^{2} \varepsilon^{*}\left(R\left(F_{2}(\xi)\right)\right)\right] d \xi-
\end{aligned}
$$




$$
\begin{aligned}
& \left.-\int_{z_{20}}^{R(t)+a_{0} t} \frac{R\left(F_{2}(\xi)\right)}{\left(\lambda_{0}+2 G_{0}\right)}\left[\sigma_{r r}^{*}\left(r_{0}\right)-\rho_{0} a_{0}^{2} \varepsilon^{*}\left(r_{0}\right)\right] d \xi+\int_{z_{20}}^{R(t)+a_{0} t} R\left(F_{2}(\xi)\right) \cdot \varepsilon^{*}\left(F_{2}(\xi)\right) d \xi\right\}+ \\
& +\frac{2}{r^{3}}-C_{24}^{-} a_{0} t+C_{35}^{+}+\int_{z_{20}}^{r+a_{0} t} d \xi_{2} \cdot \int_{z_{20}}^{\xi_{2}} d \xi_{1} \int_{z_{10}}^{\xi_{2}} \varphi(\xi) d \xi-\int_{z_{20}}^{r+a_{0} t} d \xi_{2} \int_{z_{20}}^{\xi_{2}} d \xi_{1} \int_{z_{10}}^{\left.R\left(F_{2}\left(\xi_{1}\right)\right)-a_{0} F_{2}\left(\xi_{1}\right)\right)} \varphi(\xi) d \xi+ \\
& +\int_{z_{20}}^{r+a_{0} t} d \xi_{2} \int_{z_{20}}^{\xi_{2}} \frac{R\left(F_{2}(\xi)\right)}{\left(\lambda_{0}+2 G_{0}\right)} d \xi_{1} \int_{r_{0}}^{R\left(F_{2}\left(\xi_{1}\right)\right)} Q(\xi) d(\xi)+\int_{z_{20}}^{r+a_{0} t} d \xi_{1} \int_{z_{20}}^{\xi_{1}} \frac{R\left(F_{2}\left(\xi_{1}\right)\right)}{\left(\lambda_{0}+2 G_{0}\right)} . \\
& \left.\cdot\left[\sigma_{r r}^{*}\left(r_{0}\right)-\rho_{0} a_{0}^{2} \varepsilon^{*}\left(r_{0}\right)\right] d \xi+\int_{z_{20}}^{r+a_{0} t} d \xi_{1} \int_{z_{20}}^{\xi_{1}} R\left(F_{2}(\xi)\right) \cdot \varepsilon^{*}\left(F_{2}(\xi)\right) d \xi\right\}- \\
& -\frac{2}{R^{3}(t)}\left\{-C_{24}^{-} a_{0} t+C_{35}^{+}+\int_{z_{10}}^{R(t)-a_{0} t} d \xi_{2} \int_{z_{20}}^{\xi_{2}} d \xi_{1} \int_{z_{10}}^{\xi_{1}} \phi(\xi) d \xi-\int_{z_{20}}^{R(t)+a_{0} t} d \xi_{2} \int_{z_{20}}^{\xi_{2}} d \xi_{1} \int_{z_{10}}^{\left.R\left(F_{2}\left(\xi_{1}\right)\right)-a_{0} F_{2}\left(\xi_{1}\right)\right)} \phi(\xi) d \xi+\int_{z_{20}}^{R(t)+a_{0} t} d \xi_{2} .\right. \\
& \cdot \int_{z_{20}}^{\xi_{2}} \frac{R\left(F_{2}(\xi)\right)}{\left(\lambda_{0}+2 G_{0}\right)} d \xi_{1} \int_{r_{0}}^{R\left(F_{2}\left(\xi_{1}\right)\right)} Q(\xi) d(\xi)+\int_{z_{20}}^{R(t)+a_{0} t} d \xi_{1} \int_{z_{20}}^{\xi_{1}} \frac{R\left(F_{2}(\xi)\right)}{\left(\lambda_{0}+2 G_{0}\right)}\left[\sigma_{r r}^{*}\left(r_{0}\right)-\rho_{0} a_{0}^{2} \varepsilon^{*}\left(r_{0}\right)\right] d \xi+ \\
& \left.+\int_{z_{20}}^{R(t)+a_{0} t} d \xi_{1} \int_{z_{20}}^{\xi_{1}} R\left(F_{2}(\xi)\right) \cdot \varepsilon^{*}\left(F_{2}(\xi)\right) d \xi\right\}-\frac{1}{\left(\lambda_{0}+2 G_{0}\right)}\left\{\frac{1}{3} \int_{R(t)}^{r} Q(\xi) d(\xi)+\frac{2}{3 r^{3}} \int_{r_{0}}^{r} \xi^{3} Q(\xi) d \xi-\frac{2}{3 R^{3}(t)}\right. \text {. } \\
& \left.\cdot \int_{r_{0}}^{R(t)} \xi^{3} Q(\xi) d \xi-\frac{2}{r^{3}} \int_{r_{0}}^{r} \xi^{2}\left[\sigma_{r r}^{*}(\xi)-\rho_{0} a_{0}^{2} \varepsilon^{*}(\xi)\right] d \xi+\frac{2}{R^{3}(t)} \int_{r_{0}}^{R(t)} \xi^{3}\left[\sigma_{r r}^{*}(\xi)-\rho_{0} a_{0}^{2} \varepsilon^{*}(\xi)\right] d \xi\right\}- \\
& -\frac{1}{\left(\lambda_{0}+2 G_{0}\right)}\left\{\left[\sigma_{r r}^{*}(r)-\rho_{0} a_{0}^{2} \varepsilon^{*}(r)\right]-\left[\sigma_{r r}^{*}(R(t))-\rho_{0} a_{0}^{2} \varepsilon^{*}(R(t))\right]\right\}+ \\
& +\frac{2 r^{3}}{3\left(\lambda_{0}+2 G_{0}\right)}\left(\frac{1}{r^{3}}-\frac{1}{R^{3}(t)}\right)\left[\sigma_{r r}^{*}\left(r_{0}\right)-\rho_{0} a_{0}^{2} \varepsilon^{*}\left(r_{0}\right)\right],
\end{aligned}
$$

where

$$
\begin{aligned}
& z_{10}=r_{0}-a_{0} t_{0}, z_{20}=r_{0}+a_{0} t_{0}, \\
& u_{t}(r, t(r))=-\dot{R}(t(r)) \cdot \varepsilon^{*}(r), \varepsilon_{r r}(R(t), t)=\varepsilon_{r r}^{*}(t)=\varepsilon^{*}(t), \\
& C_{35}^{+}=0, C_{24}^{-}=-\frac{r_{0}^{2} \dot{R}(0) \varepsilon^{*}(0)}{a_{0}} .
\end{aligned}
$$

Further, according to

$$
\begin{aligned}
& \sigma_{r r}=\lambda \varepsilon+2 G \varepsilon_{r r}, \quad \sigma_{\varphi \varphi}=\sigma_{00}=\lambda \varepsilon+2 G \varepsilon_{\varphi \varphi} \\
& \varepsilon=\frac{\partial u}{\partial r}+2 \frac{u}{r} ; \lambda=\frac{\sigma}{\varepsilon}-\left(\frac{2}{9}\right) \frac{\sigma_{i}}{\varepsilon_{i}} ; \quad G=\left(\frac{1}{3}\right) \frac{\sigma_{i}}{\varepsilon_{i}}
\end{aligned}
$$

and

$$
\begin{aligned}
& \sigma_{r r}=\sigma_{r r}^{*}(r)+\lambda_{0}\left[\varepsilon-\varepsilon^{*}(r)\right]+2 G\left[\varepsilon_{r r}-\varepsilon_{r r}^{*}(r)\right] \\
& \left.\sigma_{\varphi \varphi}=\sigma_{\varphi \varphi}^{*}(r)+\lambda_{0}() \varepsilon-\varepsilon^{*}(r)\right)+2 G_{0}\left(\varepsilon_{\varphi \varphi}-\varepsilon_{\varphi \varphi}^{*}(r)\right)
\end{aligned}
$$

considering (20), the volumetric deformation $\varepsilon(r, t)$ and stress components $\sigma_{r r}(r, t)$ . $\sigma_{\varphi \varphi}(r, t)$ of the soil in the disturbance region are determined (Figure 3 ). 
Substituting the expression for $\sigma_{r r}(r, t)$, obtained in the course of solving the problem, into the boundary condition

$$
\sigma_{r r}=-\sigma_{0}(t) \text { nрu } \quad r=r_{0}, t \geq 0 \text { где } u_{t}=\frac{\partial u}{\partial t}=u, \quad \dot{R}(t)=\frac{d R}{d t}
$$

we obtain a formula for determining the load profile $\sigma_{0}(t)$.

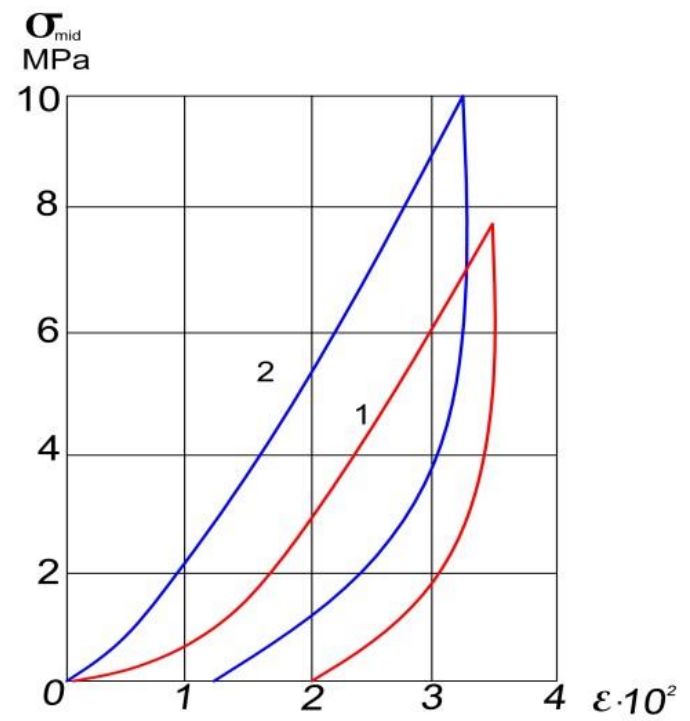

\section{$\boldsymbol{O}_{\text {mid }}$ \\ $\mathrm{MPa}$}

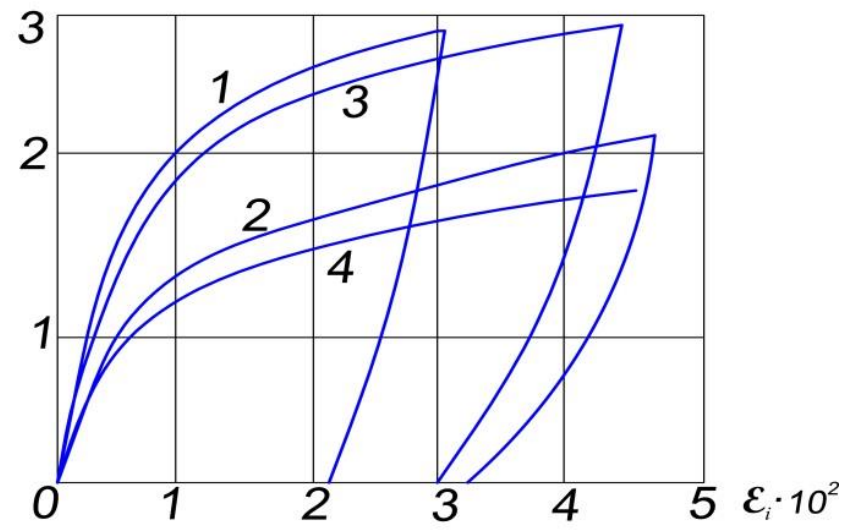

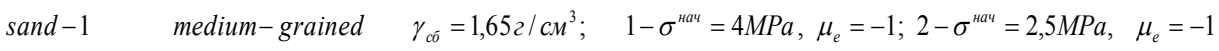
sand-2 fine-grained $\gamma_{c \tilde{\sigma}}=1,612 / \mathrm{cm}^{3} . \quad 3-\sigma^{\text {иач }}=4 M P a, \mu_{e}=+1 ; 4-\sigma^{\text {иач }}=2,5 \mathrm{MPa}, \mu_{e}=+1$

Fig. 3. Graph of the change in the shock wave in the soil $r=R(t)$ 


\section{Results and Discussion}

Specific calculations on a computer were carried out for the following initial data:

$$
\begin{gathered}
a_{1}=2.69975 \mathrm{MPa}, \boldsymbol{B}_{1}=137.3133, c_{1}=0.188206 \mathrm{MPa}, \\
a_{2}=1.24866 \mathrm{MPa}, \boldsymbol{B}_{2}=237.9847, c_{2}=15.873 \cdot 10^{3} \mathrm{MPa}, \\
E_{1}=1.4 \cdot 10^{3} \mathrm{MPa}, E_{2}=0.2 \cdot 10^{3} \mathrm{MPa}, \rho=2.0 \frac{\mathrm{kN} \cdot \mathrm{sec}^{2}}{\mathrm{~m}^{4}}, \\
r_{0}=0.1-1.0 \mathrm{M}, \quad R_{1}=420 \mathrm{~m} / \mathrm{sek}, R_{2}=2 \cdot 10^{2} R_{1}(\mathrm{~m} / \mathrm{sek})
\end{gathered}
$$

In this case, the shock front is given in the form

$$
R(t)=r_{0}+R_{1} t-R_{2} t^{2} / 2, R(t) \succ 0
$$

The calculation results are presented in Figures 4-6 for $r_{0}=0.1 \mathrm{~m}$ and in Fig.7 for $r_{0}=1.0 \mathrm{~m}$. Moreover, in Figure 4, curves 1, 2, 3 refer to sections $r=0.1 ; 0.11$ and $0.12 m$, curves 4 - to the front of a spherical shock wave $r=R(t)$, and curves 5 - to the surface $r=f(t)$ where the radial stress $\sigma_{r r}$ vanishes.

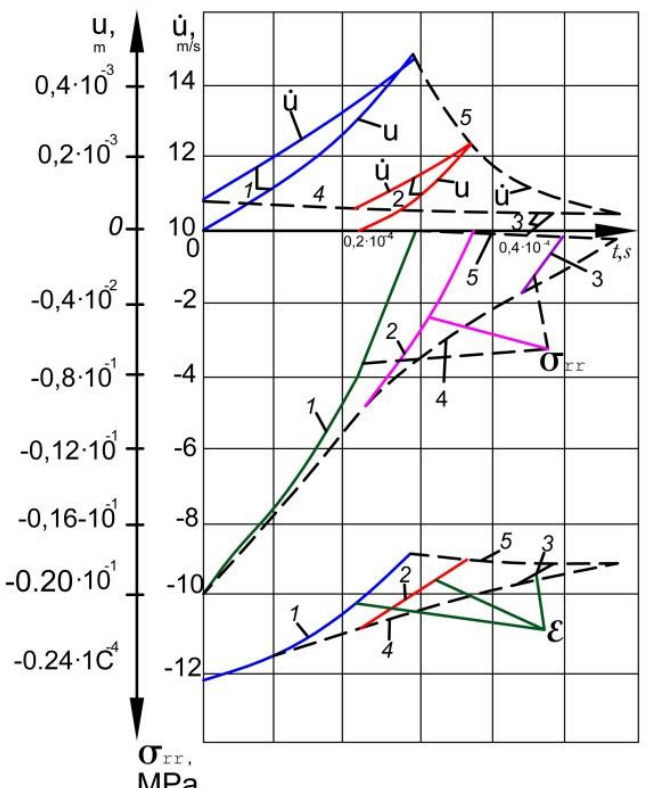

Fig. 4. Curves of changes in stress $\sigma_{r r}$, speed $\dot{u}$, volumetric deformation $\varepsilon$ and displacement $u(r, t)$ depending on time $t$ at (curves 1,2 3), where curves 4 and 5 correspond to the front of the wave $r=R(t)$ and surface $r=f(t)$, where $\sigma_{r r}=0$ 
Figure 5 shows that surface $r=f(t)$, where $\sigma_{r r}=0$, turns out to be elongated towards the front of the shock wave, and the load $\sigma_{0}(t)$ is concave to the axis $o t$. The speed of the shock wave $R$, depending on the coordinate $r$, linearly decays (Figure 6).

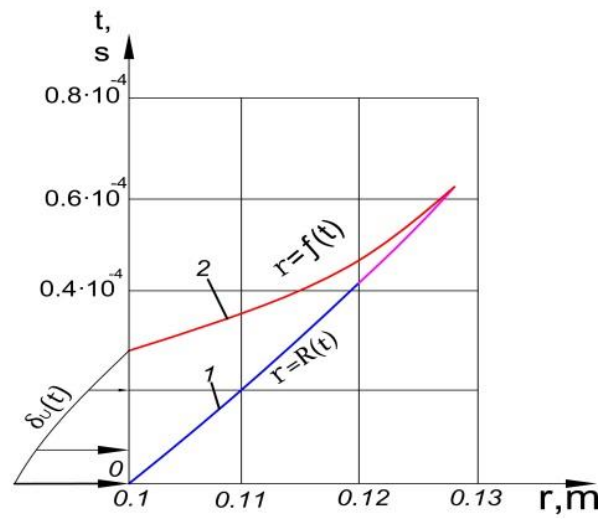

Fig. 5. Change in the load profile $\sigma_{0}(t)$ on the cavity with radius $r_{0}=0,1 \mathrm{~m}$ and surface shape $r=f(t)$, where $\sigma_{r r} \equiv 0$, depending on time $t$.

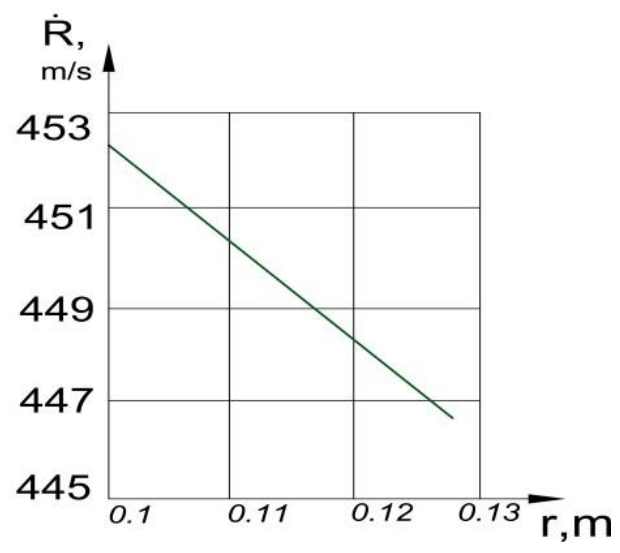

Fig. 6. Change in the speed of the front of a spherical wave $\dot{R}$ depending on the distance $r$

The calculation results show that the load profiles $\sigma_{0}(t)=-\sigma_{r r}\left(r_{0}, t\right)$ obtained using the inverse method for cases (3) (Fig.7 dashed lines) and (3) (Fig.7 solid lines) are significantly different and have a decaying character depending on the time $t$. In this case, the $\sigma_{0}(t)$ curve for (3) is located higher in absolute value than the $\sigma_{0}(t)$ curve for (1). In the latter case, the law of decay of the curve $\sigma_{0}(t)$ turns out to be steeper with the smallest time interval of action on the boundary of the spherical cavity than for (3). In contrast to case (3) at (1), the mass velocity $\dot{u}$ of the soil gradually increases depending on the time T (Fig. 7). The radial stress $\sigma_{r r}$ at the cavity boundary decreases with time faster than at the front of the spherical wave $r=R(t)$, (Figure 4 curves 1-4). 


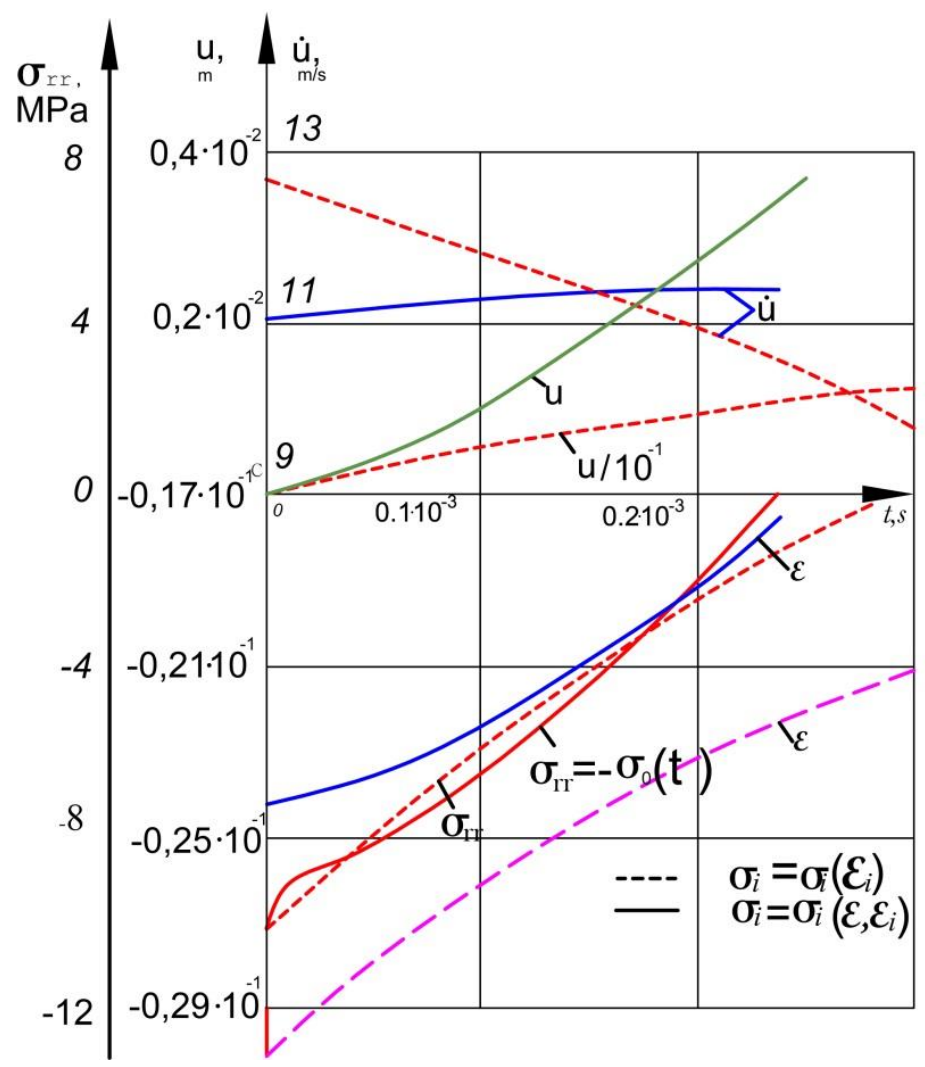

Fig. 7. Change in stress $\sigma_{r r}$, mass velocity $\dot{u}$, volumetric deformation ${ }^{\varepsilon}$ and displacement $u(r, t)$ on a spherical cavity of radius $r_{0}=0.1 \mathrm{~m}$ depending on time ${ }^{t}$ for cases $\sigma_{i}=\sigma_{i}\left(\varepsilon, \varepsilon_{i}\right)_{\text {(solid) and }} \sigma_{i}=\sigma_{i}\left(\varepsilon_{i}\right)_{\text {(dotted lines) }}$

Thus, we note that in the case of using the complicated equation of state of the soil (1), a spherical shock wave $r=R(t)$ propagates in the soil, behind the front of which, in the disturbance region, the medium is unloaded. Similarly, you can research the case when $\sigma=\sigma\left(\varepsilon, \varepsilon_{i}\right)$.

\section{Conclusions}

1. The problem of the propagation of a spherical wave in the soil is solved analytically in the opposite way based on the deformation theory, taking into account the generalized equations of the state of the medium.

2. The results are obtained by the inverse method of propagation of spherical shock waves in soil with complex equations of state. Concrete calculations on a computer show that the curves to the front of the spherical shock wave $r=R(t)$, and the curves to the surface $r=f(t)$, where the radial stress $\sigma_{r r}$ vanishes. 
3. It was found that in the case of using the complicated equation of state (1), a spherical shock wave $r=R(t)$ propagates in the soil, behind the front of which, in the perturbed region, the medium is unloaded.

4. The calculation results show that the load profiles $\sigma_{0}(t)$ obtained using the inverse method for cases (1) (Figures 1, 7 dashed lines) and (1.2) (Figures 1, 7 solid lines) are significantly different and have a decaying character depending on the time $t$.

5. In the process of solving the problem, it is assumed that when using $(1,4)$ and $(1)$, a spherical shock wave propagates in the soil, at the front of which the medium is instantly loaded in a nonlinear manner, followed by a linear irreversible unloading with Young's modul $E_{1}$ and $E_{2}$.

\section{References}

1. Liao, Y., Mahardika, N., Zhao, X., Lee, J., He, J. Shock wave propagation in long laboratory sparks under negative switching impulses. Journal of Physics D: Applied Physics 54(1), (2021), № 015205. DOI: 10.1088/1361-6463/abb8ff.

2. Winter, K.O., Hargather, M.J. Three-dimensional shock wave reconstruction using multiple high-speed digital cameras and background-oriented schlieren imaging. Exp Fluids 60, 93 (2019). https://doi.org/10.1007/s00348-019-2738-x.

3. Tomar, A., Arora, R., Chauhan, A. Propagation of strong shock waves in a non-ideal gas. Acta Astronautica, 159 (2019), pp. 96-104.

4. Paul, R.A., Forbes, L.K. A reacting shock in a spherically symmetric gas. Journal of Engineering Mathematics. 113(1), 2018. DOI: 10.1007/s10665-018-9970-x.

5. Nath, G., Vishwakarma, J.P. Propagation of a strong spherical shock wave in a gravitating or non-gravitating dusty gas with exponentially varying density. Acta Astronautica. 123(1), 2016, pp. 200-212. DOI: 10.1016/j.actaastro.2016.03.009.

6. Steiner, H., Gretler, W. The propagation of spherical and cylindrical shock waves in real gases. Physics of Fluids. 6(6), (1994), pp. 2154-2164. DOI: 10.1063/1.868218.

7. Sultanov, K.S. The attenuation of longitudinal waves in non-linear viscoelastic media // Journal of Applied Mathematics and Mechanics. 2002. 66(1). pp. 115-122. DOI:10.1016/S0021-8928(02)00015-1.

8. Sultanov, K.S., Kumakov, J.X., Loginov, P. V., Rikhsieva, B.B. Strength of underground pipelines under seismic effects // Magazine of Civil Engineering. 2020. 93(1). pp. 97-120.

9. Bakhodirov, A.A., Ismailova, S.I., Sultanov, K.S. Dynamic deformation of the contact layer when there is shear interaction between a body and the soil // Journal of Applied Mathematics and Mechanics. 2015. 79(6). pp. 587-595. DOI:10.1016/j.jappmathmech.2016.04.005

10. Sultanov, K.S. A non-linear law of the deformation of soft soils // Journal of Applied Mathematics and Mechanics. 1998. 62(3). pp. 465-472. DOI:10.1016/S00218928(98)00058-6.

11. Sultanov, K.S., Bakhodirov, A.A. Laws of Shear Interaction at Contact Surfaces Between Solid Bodies and Soil // Soil Mechanics and Foundation Engineering. 2016. 53(2). pp. 71-77. DOI:10.1007/s11204-016-9367-7

12. Mirsaidov, M.M., Khudainazarov, Sh.O. Spatial natural vibrations of viscoelastic axisymmetric structures. Magazine of Civil Engineering. No. 04. 2020. 96(4). pp. 118-128. DOI: 10.18720/MCE.96.10

13. Khudainazarov, Sh.O., Donayev, B., Yarashov, J.A. Non-stationary oscillations of high-rise axisymmetric structures. IOP Conf. Series: Materials Science and Engineering 883 (2020) 012195. doi:10.1088/1757-899X/883/1/012195 
14. Khudainazarov, Sh.O., Mavlanov, T., Qosimov, J., Nurova, O.S. Forced vibrations of high-rise buildings. IOP Conf. Series: Materials Science and Engineering 869, (2020), pp.1-13. doi:10.1088/1757-899X/869/5/052047.

15. Mirsaidov, M.M., Abdikarimov, R., Khudainazarov, Sh.O., Sabirjanov, T. Damping of high-rise structure vibrations with viscoelastic dynamic dampers. E3S Web of Conferences 224, 02020 (2020) TPACEE-2020. pp. 1-14

16. Khudainazarov, Sh.O., Donayev, B., Abdimuminov, E., Suyunova, Y. Interaction of shock waves with elastic-plastic medium. IOP Conf. Series: Materials Science and Engineering 869 (2020) 052074

17. Mirsaidov, M.M., Sultanov, T.Z., Rumi, D.F. An assessment of dynamic behavior of the system "structure - Foundation" with account of wave removal of energy. Magazine of Civil Engineering. 2013. 39(4), pp. 94-105. DOI: 10.5862/MCE.39.10.

18. Sultanov, T.Z., Khodzhaev, D.A., Mirsaidov, M.M. The assessment of dynamic behavior of heterogeneous systems taking into account non-linear viscoelastic properties of soil. Magazine of Civil Engineering. 2014. 45(1), pp. 80-89+117-118. DOI: 10.5862/MCE.45.9.

19. Mirsaidov, M. An account of the foundation in assessment of earth structure dynamics. 2019. E3S Web of Conferences. 97,04015. DOI: $10.1051 / \mathrm{e} 3 \operatorname{sconf} / 20199704015$.

20. Jiang, J., Blair, D. P., Baird, G. R. Dynamic response of an elastic and viscoelastic full-space to a spherical source International Journal for Numerical and Analytical Methods in Geomechanics.Vol. 19, Issue 3. pp 181-193. DOI: $10.1002 /$ nag. 1610190303 .

21. Chattopadhyay, A., Michel, V.A. Model for spherical SH wave propagation in selfreinforced linearly elastic media Archive of Applied Mechanics vol.75 pp.113-124 https://doi.org/10.1007/s00419-005-0417-2.

22. Safarov, I.I., Teshaev, M.K, Boltaev, Z.I Propagation of linear waves in multilayered structural - Inhomogeneous cylindrical shells. Journal of Critical Reviews, Volume 7, Issue 12, 2020, Pages 893-904. DOI: 10.31838/jcr.07.12.157.

23. Safarov, I.I., Teshaev, M., Toshmatov, E., Boltaev, Z., Homidov, F. Torsional vibrations of a cylindrical shell in a linear viscoelastic medium. IOP Conference Series: Materials Science and Engineering 883(1), 2020, № 0121902020, DOI: $10.1088 / 1757-899 \mathrm{X} / 883 / 1 / 012190$.

24. Rakhmatulin, Kh.A., Sagomonyan, A.Ya., Alekseev, N.A. Issues of soil dynamics. M.: Publishing House of Moscow State University, p.239, (1964)

25. Rakhmatullin Kh.A. On the propagation of elastic-plastic waves owing to combined loading. Journal of Applied Mathematics and Mechanics. 22(6), 1958, pp. 1079-1088, DOI: 10.1016/0021-8928(58)90034-0.

26. Investigation of the mechanical properties of soils under conditions of triaxial compression at an elevated level of stress. Report of the Moscow Institute of Mathematics and Mathematics named after V.V. Kuybyshev, No. 320 M., p.68, (1972) 